\title{
Low-Conductance HCN1 Ion Channels Augment the Frequency Response of Rod and Cone Photoreceptors
}

\author{
Andrew J. Barrow ${ }^{1,2}$ and Samuel M. Wu ${ }^{2}$ \\ ${ }^{1}$ Department of Bioengineering, Rice University, Houston, Texas 77005, and ${ }^{2}$ Cullen Eye Institute, Baylor College of Medicine, Houston, Texas 77030
}

\begin{abstract}
Hyperpolarization-activated cyclic nucleotide-gated $(\mathrm{HCN})$ ion channels are expressed in several tissues throughout the body, including the heart, the CNS, and the retina. HCN channels are found in many neurons in the retina, but their most established role is in generating the hyperpolarization-activated current, $I_{\mathrm{h}}$, in photoreceptors. This current makes the light response of rod and cone photoreceptors more transient, an effect similar to that of a high-pass filter. A unique property of HCN channels is their small single-channel current, which is below the thermal noise threshold of measuring electronics. We use nonstationary fluctuation analysis (NSFA) in the intact retina to estimate the conductance of single $\mathrm{HCN}$ channels, revealing a conductance of $\sim 650 \mathrm{fS}$ in both rod and cone photoreceptors. We also analyze the properties of HCN channels in salamander rods and cones, from the biophysical to the functional level, showing that $\mathrm{HCN} 1$ is the predominant isoform in both cells, and demonstrate how HCN1 channels speed up the light response of both rods and cones under distinct adaptational conditions. We show that in rods and cones, HCN channels increase the natural frequency response of single cells by modifying the photocurrent input, which is limited in its frequency response by the speed of a molecular signaling cascade. In doing so, HCN channels form the first of several systems in the retina that augment the speed of the visual response, allowing an animal to perceive visual stimuli that change more quickly than the underlying photocurrent.
\end{abstract}

\section{Introduction}

Hyperpolarization-activated cyclic nucleotide-gated (HCN) ion channels are activated by membrane hyperpolarization, and depending on the channel isoform, modulated by cyclic nucleotides such as cAMP to a varying degree (Baruscotti et al., 2005; DiFrancesco, 2006; Siu et al., 2006). HCN channels serve important functions in many systems of the body. For example, they act as a cardiac pacemaker in the SA node and other parts of the heart (DiFrancesco, 2006) and contribute to oscillatory potentials in the CNS (Siu et al., 2006). In the retina, immunocytochemical studies have shown that various isoforms of HCN channels are expressed in retinal neurons (Müller et al., 2003), but the functional specificity of these expression patterns are not clear. In rod and cone photoreceptors, hyperpolarization-activated currents $\left(I_{\mathrm{h}}\right)$ have been characterized with whole-cell or microelectrode recording techniques (Hestrin, 1987; Barnes and Hille, 1989; Maricq and Korenbrot, 1990; Demontis et al., 1999; Demontis et al., 2002). It is not clear, however, whether $I_{\mathrm{h}}$ in rods and cones is mediated by HCN channels and whether these two photoreceptors use the same isoform of channels. Although current-voltage relations and activation properties of whole-cell $I_{\mathrm{h}}$ currents in rods have been described (Hestrin, 1987; Demontis et al., 1999, 2002), the single-channel conductance and density of $I_{\mathrm{h}}$ or HCN

Received Dec. 1, 2008; revised Feb. 23, 2009; accepted Feb. 28, 2009.

This work was supported by grants from National Institutes of Health (NIH) (EY 04446), NIH Vision Core (EY 02520), the Retina Research Foundation, and Research to Prevent Blindness, Inc. We thank Roy Jacoby, Cameron Cowan, and David Simons for critically reading this manuscript.

Correspondence should be addressed to Andrew Barrow, Cullen Eye Institute, Baylor College of Medicine, 6565 Fannin, NC 420, Houston, TX 77030. E-mail: andrew.barrow@rice.edu.

DOI:10.1523/JNEUROSCI.5746-08.2009

Copyright $\odot 2009$ Society for Neuroscience $\quad$ 0270-6474/09/295841-13\$15.00/0 channels in photoreceptors are unknown. Here we present a systematic study of the single-channel properties of HCN channels in salamander rod and cone photoreceptors using the nonstationary fluctuation analysis (NSFA) method. By comparing single-channel conductance with whole-cell currents, we estimate the total number of channels and the channel density in rods and cones. We also demonstrate via immunohistochemistry, single-channel conductance, and analysis of kinetic data of whole-cell currents that $I_{\mathrm{h}}$ in salamander rods and cones is mediated by the $\mathrm{HCN} 1$ isoform.

In contrast to the heart and CNS, where HCN channels generate rhythmic potentials, HCN channels in the retina do not cause oscillations, but instead help shape the potentials that encode light stimuli. Evidence suggests that HCN channels are necessary for the retina's temporal response to light stimuli (Gargini et al., 1999a,b,c). The $I_{\mathrm{h}}$ current, along with another ionic conductance dubbed $I_{\mathrm{Kx}}$, have been shown to create a bandpass filter effect in rod photoreceptors (Attwell and Wilson, 1980; Detwiler et al., 1980; Owen and Torre, 1983; Torre and Owen, 1983; Baylor et al., 1984; Beech and Barnes, 1989; Demontis et al., 1999). It is not clear, however, how the electrical properties of HCN channels contribute to the dynamics of rod and cone signaling, or whether these processes differ in the two types of photoreceptors. In this study, we use frequency-chirped and Gaussian white noise (GWN)-modulated current and light stimuli to directly demonstrate how HCN channels speed up the voltage response of rods and cones. Our results show that HCN channels contribute to photoreceptor dynamics not only in response to bright flashes, where signal clipping is a factor, but also under physiologic light levels, and have varying degrees of effect in rods and cones at different adaptational conditions. 


\section{Materials and Methods}

Tiger salamanders (Ambystoma tigrinum) were kept on a $12 \mathrm{~h}$ light-dark cycle in a temperature-controlled environment. Animals were handled according to National Institutes of Health guidelines, and the Baylor College of Medicine Committee for Animal Use approved the methods of this study. The salamanders were first dark adapted for $45 \mathrm{~min}$, then anesthetized with MS2222, and quickly decapitated after they were unresponsive. Eyes were then enucleated and the retinas were dissected under infrared light with night vision scopes (BE Meyers) mounted to a stereomicroscope. The dissected whole retina was then fixed to a piece of filter paper which was first secured to the bottom of the recording chamber with a small amount of silicon grease and with a small window for transillumination carved into it. The ground electrode consisted of chlorided silver wire. The chamber was mounted in a Zeiss Universal Microscope (Carl Zeiss) with Hoffman Modulation Contrast inside a lightisolating Faraday cage. Perfusion was supplied by gravity feed through a valve manifold (ALA Scientific), and solution was recirculated with a peristaltic pump. Extracellular solutions were bubbled with $100 \% \mathrm{O}_{2}$. For experiments measuring light responses, a deep infrared filter was placed on the microscope condenser, and all extraneous light sources were eliminated. Night vision scopes mounted to the microscope eyepieces converted infrared light to visible light to view the preparation. An MP-285 micromanipulator (Sutter Instruments) provided fine control of the pipette electrode for recordings.

\section{Solutions}

Normal Ringer's solution was used for recordings of cell light response, and consisted of (in mM) $108 \mathrm{NaCl}, 2.5 \mathrm{KCl}, 1.2 \mathrm{MgCl}_{2}, 2 \mathrm{CaCl}_{2}$, and 5 HEPES. Solution $\mathrm{pH}$ was titrated to 7.7 with $\mathrm{NaOH}$. For recordings of $\mathrm{HCN}$-gated currents, $20 \mathrm{TEA}, 5 \mathrm{CoCl}_{2}$, and $5 \mathrm{BaCl}_{2}$ were added to the solution to block BK and Ca-dependent potassium channels, calcium channels, and $I_{\mathrm{Kx}}$ potassium channels, respectively. Intracellular solutions for whole-cell recordings consisted of (in mM) $106 \mathrm{~K}$ gluconate, 5 $\mathrm{NaCl}, 2 \mathrm{MgCl}_{2}, 5 \mathrm{EGTA}$, and 5 HEPES, with pH 7.4 titrated with $\mathrm{KOH}$. Pipette solutions for cell-attached recordings of HCN channels consisted of $106 \mathrm{KCl}, 167 \mathrm{NaCl}, 1.8 \mathrm{CaCl}_{2}, 1 \mathrm{MgCl}_{2}$, and 5 HEPES, and were titrated to $\mathrm{pH} 7.7$ with $\mathrm{NaOH}$. The purpose of this nonphysiologic solution was to increase the driving force of the HCN channels inside the patch. The reversal potential for the HCN channels with this solution was approximately $+9 \mathrm{mV}$, assuming a $[\mathrm{Na}] /[\mathrm{K}]$ permeability ratio of 0.33 (Hestrin, 1987; Wollmuth and Hille, 1992). In chirped current and current step injection experiments, $\mathrm{K}^{+}$and $\mathrm{Ca}^{2+}$ channels were blocked, and the HCN channel blocker ZD 7288 was perfused at a concentration of 100 $\mu \mathrm{M}$ to selectively block the $I_{\mathrm{h}}$ current. Although ZD 7288 is known to be a specific blocker of HCN channels (Baruscotti et al., 2005), in light response experiments, normal saline solution was used, and the ZD 7288 concentration was reduced to $50 \mu \mathrm{M}$ to minimize potential crossover to other ion channels.

\section{Recordings}

Recordings were made with an EPC-10 amplifier (HEKA) in either voltage-clamp or current-clamp mode. All power supply cables were shielded, which along with the Faraday cage were well grounded (Hamill et al., 1981). Baseline noise levels recorded in voltage-clamp mode with no pipette attached to the pipette holder were measured to be $140 \mathrm{fA}$ RMS. Unless otherwise stated, data were low-pass filtered at $4 \mathrm{kHz}$ with a Bessel filter, and sampled at $10 \mathrm{kHz}$ to avoid aliasing. Patchmaster software (HEKA) was used to generate stimulus pulses and record the data.

Glass electrodes were pulled from $1.5 \mathrm{~mm}$ OD thick-walled $(0.86 \mathrm{~mm}$ ID) $10 \mathrm{~cm}$ glass with filament (Sutter Instruments) on a programmable P-97 puller (Sutter Instruments). Using thick glass reduced pipette capacitance and noise (Hamill et al., 1981). Smaller-tipped pipettes were pulled for whole-cell recording to minimize the effects of washout. These pipettes measured 8-10 $\mathrm{M} \Omega$ when filled with intracellular solution. These results are corrected for a calculated liquid junction potential of +14 mV (Sakmann and Neher, 1995). Larger-tipped pipettes were pulled for cell-attached recordings to increase the number of HCN channels present in a patch. These pipettes measured between 1.5-3 $\mathrm{M} \Omega$ when filled with the cell-attached solution. Gigaseals with these pipettes incor- porated a large area of membrane to maximize the HCN channel current. Typical cell-attached background noise levels with a gigaseal patch were 400-600 fA RMS at $4 \mathrm{kHz}$ bandwidth.

To measure the $I_{\mathrm{h}} I-V$ curve, activation curve, and activation time constants, a voltage-clamp protocol that pulsed cells from a holding potential to command potentials between $-134 \mathrm{mV}$ and $-24 \mathrm{mV}$ and then to a tail potential of $-4 \mathrm{mV}$ was used. Leak currents were subtracted with a standard P/4 protocol (Sakmann and Neher, 1995).

For chirped current stimulus recordings, stimuli were modulated by exponentially increasing frequencies (Eq. 1) to increase the signal component in the low-frequency range, where the response of the cell is more interesting. Frequency range was $0.5-20 \mathrm{~Hz}$, and delivered over a period of $20 \mathrm{~s}$. MATLAB (MathWorks) was used to generate frequency-chirped sine wave stimuli (Eq. 1) and Gaussian white noise stimuli, which were then fed into Patchmaster. Frequency-chirped stimuli were sampled at $500 \mathrm{~Hz}$. Current amplitudes were $\sim 50 \mathrm{pA}$ with a $50 \mathrm{pA}$ offset, but were determined at the time of experiment by the voltage response to steps of current input. Stimuli were calibrated to have voltage responses no less than $-100 \mathrm{mV}$ to avoid dielectric breakdown.

$$
I_{\text {stim }}=I_{\text {amp }} \cdot \cos \left(2 \pi f_{\text {start }} \cdot e^{(t \cdot k)} \cdot t\right)+I_{\text {offset }}
$$

Recordings of whole-cell currents from both rods and cones were made using the whole-mount retina. To control for the potential effect of $I_{\mathrm{h}}$ activation in adjacent cells contributing to the recorded response, some recordings were made from single dissociated cells, and the results were compared against the data from the whole-mount retina. These responses cells fell within the range for recordings from the whole-mount retina (Fig. 1 A2). Although rod-rod coupling appears to play an important role in propagating small electrical signals between adjacent rods, it does not affect our characterization of the kinetics of the $I_{\mathrm{h}}$ current in a single cell. Our experiments involve much larger currents, making the contribution from neighboring cells negligible compared with the current induced in the recorded cell. Given a rod impedance of $550 \mathrm{M} \Omega$ and a coupling resistance of $2 \mathrm{G} \Omega$ (Zhang and $\mathrm{Wu}, 2005$ ), this would result in the adjacent cell being hyperpolarized at most to $-57 \mathrm{mV}$ in our experiments, where a proportionally small $I_{\mathrm{h}}$ current is generated (Fig. $1 A 3, B 3)$. Only part of this activated current in adjacent cells would flow back into the voltage-clamped cell.

\section{Light responses}

Light responses were recorded using current-clamp mode. $R_{\mathrm{s}}$ compensation in the EPC-10 acted as a bridge to ensure that the voltages were properly scaled. Light stimuli were generated by converting the voltage from the D/A converter on the amplifier into a current source via an op-amp, which was used to drive either a $627 \mathrm{~nm}$ or a $530 \mathrm{~nm}$ Luxeon $\mathrm{K} 2$ LED (Phillips). The light output from the LEDs was found to be proportional to the drive current.

\section{Impulse responses}

We estimated the impulse response of photoreceptors using the 627 or $530 \mathrm{~nm}$ LED light source modulated with $30 \mathrm{~Hz}$ Gaussian white noise. The noise was generated in MATLAB, had equal variance and mean, and was delivered to the preparation at $1000 \mathrm{~Hz}$ sample rate in phase with the current-clamp recordings. First-order kernels were estimated using the Lee-Shetzen cross-correlation approach, with the first-order kernel equal to the weighted cross-correlation between the stimulus and recorded response (Marmarelis and Marmarelis, 1978). For the purposes of this paper, we use the term impulse response to describe the first-order kernel. This is because photoreceptors can be approximated as linear-the power of the photoreceptor response is comparable to the power of the first-order kernel predicted response (Marmarelis and Naka, 1973).

\section{Immunohistochemistry}

Immunohistochemical experiments were performed using a rabbit antirat polyclonal $\mathrm{HCN} 1$ antibody (Sigma) and a rabbit anti-human HCN3 antibody (Santa Cruz Biotechnology). Salamander retina was embedded in low-temperature gelling agar (Sigma) and cut into $40 \mu \mathrm{m}$ sections with a vibratome. Sections were permeabilized and blocked with a $10 \%$ donkey serum in PBS with Triton overnight and then incubated in pri- 

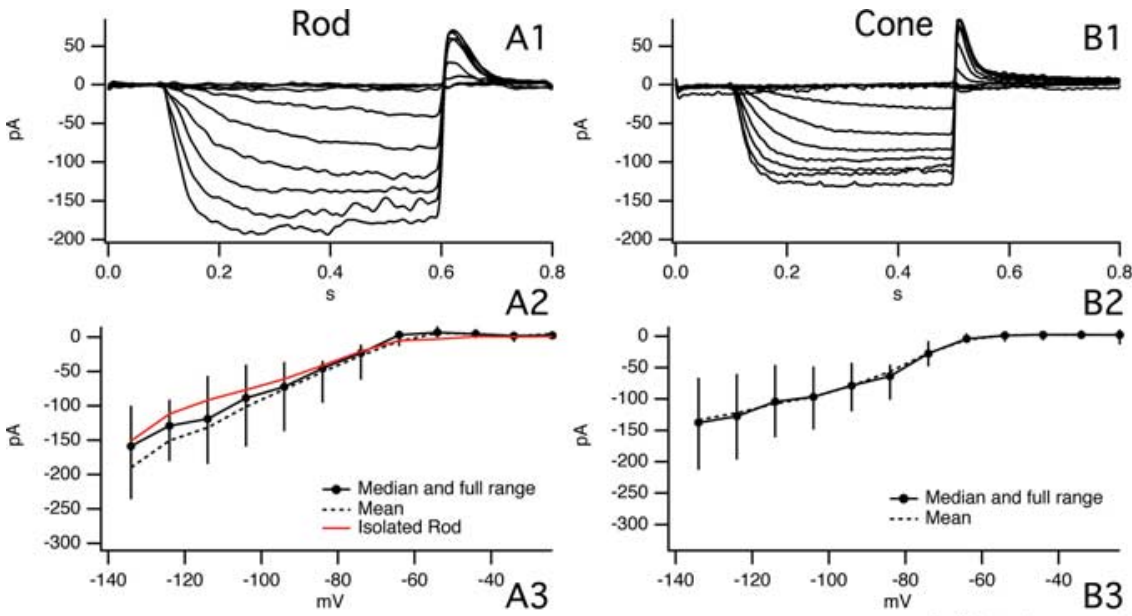

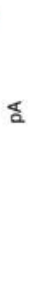
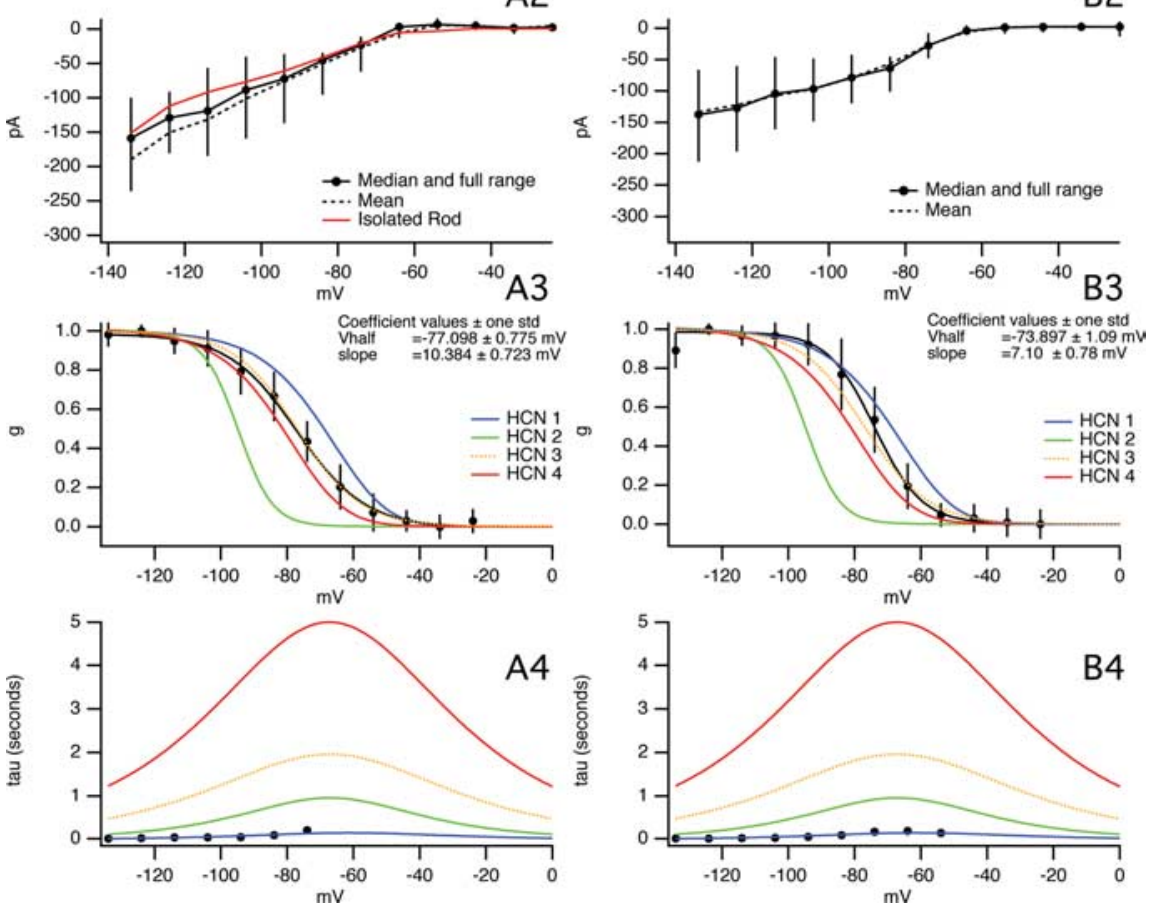

Figure 1. Whole-cell recording data from a $\operatorname{rod}(\boldsymbol{A})$ and cone $(\boldsymbol{B})$. $\boldsymbol{A} \mathbf{1}$ and $\boldsymbol{B} 1$ show example trace families for the $I-V$ protocol. $A \mathbf{2}$ and $B \mathbf{2}$ show the median $I-V$ curve values with vertical bars representing the minimum and maximum range for 10 cells. $A \mathbf{3}$ and $\boldsymbol{B} 3$ show the respective activation curves computed from the normalized and averaged tail currents of 10 cells. Data are fit with a sigmoidal exponential function of the following form: $g=b+g_{\max } /\left(1+e^{\left(V-V_{\text {half }}\right) / \text { slope }}\right) . A 4$ and $B 4$ show the activation time constants fit with a single exponential for rod $(\boldsymbol{A} 4)$ and cone (B4) data (black dots), with the color lines showing values for $\mathrm{HCN} 1, \mathrm{HCN} 2$, HCN3, and HCN4. In $A 3, A 34, B 3$, and $B 4$, data for HCN1, HCN2, and HCN4 come from homogeneously expressed HCN channels (Altomare et al., 2001) plus estimated values for expressed HCN3 (Stieber et al., 2005).

mary antibody in 3\% donkey serum PBS for $5 \mathrm{~d}$. The sections were washed and incubated overnight in donkey anti-goat antibodies tagged with Alexa-488 (Invitrogen). They were then mounted on slides and imaged on a Zeiss LSM 510 microscope.

\section{Western blot}

Western blot experiments were performed using the same HCN1 and $\mathrm{HCN} 3$ antibodies as the immunohistochemistry. The anti-HCN1 antibody's epitope was residues 6-24 (near the N terminus) of rat HCN1 protein, representing the intracellular domain. A BLASTp search in PubMed demonstrated that this sequence is highly conserved among mouse, rat, human, rabbit, and cat, and that it should not cross-react with other known proteins. The anti-HCN3 antibody's epitope was amino acids 625-774 near the $\mathrm{C}$ terminus of the human HCN3 protein.

Mouse brain and 12 salamander retinas were extracted and homogenized in a buffer consisting of $500 \mathrm{~mm} \mathrm{NaCl}, 20 \mathrm{~mm}$ Tris- $\mathrm{HCl}$ buffer at $\mathrm{pH}$ 7.5 (Bio-Rad), 2 mм EGTA (Fluka), and a protease inhibitor cocktail tablet (Roche). Extracts were kept on ice, and centrifuged at $4^{\circ} \mathrm{C}$ at $500 \times$ $g$ for $20 \mathrm{~min}$. The supernatant was extracted and pellet discarded. For the mouse brain, to isolate membrane proteins, the extract was centrifuged at $30,000 \times g$ for $20 \mathrm{~min}$ at $4^{\circ} \mathrm{C}$, and the pellet was collected. Runs of nonultracentrifuged brain extract gave identical results to the ultracentrifuged extract. Ultracentrifuged retina did not yield enough protein pellet to stain. Ultracentrifuged brain was resuspended in 10\% SDS. Extracts were combined 1:1 into a Laemmli buffer (Bio-Rad) and denatured at $80^{\circ} \mathrm{C}$ for $10 \mathrm{~min}$. Solutions were run in a $7.5 \%$ Tris Ready-gel (Bio-Rad) for $45 \mathrm{~min}$ at $100 \mathrm{~V}$, and transferred for $60 \mathrm{~min}$ at $110 \mathrm{~V}$ to a PVDF membrane presoaked in methanol. Transfer buffer was Tris/glycine $+20 \%$ methanol.

The membranes were incubated in antibodies at a 1:200 dilution in 3\% milk overnight. After three sets of washes, they were incubated once again with HRP-conjugated anti-rabbit antibody at a 1:500 dilution. Membranes were washed three times again, and reacted with ECP detection solution. The reaction was exposed onto $\mathrm{x}$-ray film for either 5 or $10 \mathrm{~min}$ and developed. BLASTp searches on antibody epitope were performed on Xenopus tropicalis data obtained from Xenbase.org (Bowes et al., 2008).

\section{Data analysis}

Data analysis was performed with IgorPro or MATLAB using custom-written and standard built-in routines.

NSFA, sometimes called nonstationary noise analysis, was used to estimate the conductance of single HCN channels (Sigworth, 1980; Johnston and Wu, 1995; Sakmann and Neher, 1995). This technique uses many repeated command voltage pulses to create a collection of many current responses. This collection of traces is referred to as an ensemble. From this ensemble, the variance and mean current can be computed at each point in time using Equation 2. A plot of the variance versus the mean was used to estimate the single-channel conductance by fitting with a parabola. We use software routines written in IgorPro for the NSFA. For our experiments, NSFA was performed on a series of 100 or more data traces pulsed to no more than -94 or $-104 \mathrm{mV}$ to avoid dielectric breakdown of the membrane. Ideally the noise analysis should be performed using a command pulse that can both fully activate the ion channel of interest and generate sufficient driving force for the current. However, photoreceptor cell membranes experience dielectric breakdown beginning near $-100 \mathrm{mV}$ (Fesenko et al., 1985). Dielectric breakdown manifests itself as a transient high amplitude $1 / f$ noise process, invalidating the fluctuation analysis in traces in which it is present. Therefore we had to use a less hyperpolarized voltage to achieve recordings uncorrupted by membrane breakdown. As a result, our NSFA estimates could achieve a maximum $P_{\mathrm{o}}=0.95 / 0.85$ for $V_{\mathrm{c}}=-104 /-94 \mathrm{mV}$.

For most recordings, Equation 2 was sufficient to estimate the variance in the current (Alvarez et al., 2002; Kole et al., 2006; Hartveit and Veruki, 2007).

$$
\begin{gathered}
k=\ln \left(\frac{f_{\text {end }}}{f_{\text {start }}}\right) / t_{\text {max }} \\
\sigma^{2}(t)=\frac{1}{N-1} \sum_{x=1}^{N}\left(i_{x}(t)-I(t)\right)^{2},
\end{gathered}
$$

where $i_{x}(\mathrm{t})$ is the trace and the mean, $I(t)$, is defined by the following:

$$
I(t)=\frac{1}{N} \sum_{x=1}^{N} i_{x}(t) .
$$

An isochrone is defined as a cross section of the ensemble of traces with respect to one point in time: 


$$
\left\{i_{x}(\tau): 1 \leq x \leq N\right\},
$$

where $\tau$ is a particular time of interest that defines the isochrone.

However, for the whole-cell NSFA estimates and some cell-attached NSFA estimates, a method that could account for the timedependent "rundown" in the current traces in the ensemble was necessary (Alvarez et al., 2002). Rundown is noticeable when the amplitude of HCN activation current or cell capacitance drifts over time, and successive traces in the ensemble are then no longer stationary with respect to their isochrone. This change could be due to a washout of cell cytoplasm, a change in membrane capacitance, or deactivation of some $\mathrm{HCN}$ channels over time. To minimize the effect of rundown, we calculated the variance from the differences between the traces, as in Equation 5 (Alvarez et al., 2002; Kole et al., 2006).

$$
\sigma^{2}(t)=\frac{2}{N-1} \sum_{x=1}^{N}\left(y_{x}(t)-Y(t)\right)^{2},
$$

with $y_{x}(\mathrm{t})$, the difference between two successive traces, defined by the following:

$$
y_{x}(t)=\frac{y_{x}-y_{x+1}}{2}
$$

and the mean of differences calculated by the following:

$$
Y(t)=\frac{1}{N-1} \sum_{x=1}^{N} y_{x}(t) .
$$

Traces that had spurious transient noise, caused by temporary membrane breakdown, external electrical interference, or other causes needed to be removed from the ensemble to generate a reliable estimate of the variance. If a minority of traces needed to be removed, and the effect of rundown was small over the ensemble, then the variance estimate according to Equation 5 was reliable. Typically, traces which had $0.1 \%$ of their points 3 or more SDs from the mean were removed. All results in this paper are reported as mean \pm SEM unless otherwise indicated.

After acquisition at $10 \mathrm{kHz}$ and filtering with a low-pass Bessel filter at $4 \mathrm{kHz}$, data were imported into IgorPro for processing, and digitally filtered to $400 \mathrm{~Hz}$ with a Gaussian weighted FIR filter. This additional filtering step allowed us to increase the signal-to-noise ratio of our recordings by reducing broad spectrum background noise fluctuations. Theoretical considerations dictate that the filter time constant should be no more than 10 times the activation time constant of the recorded channels (Alvarez et al., 2002). HCN channels were seen to transition with a time constant of $\sim 50 \mathrm{~ms}$ at $-104 \mathrm{mV}$, which would yield a minimum filter time constant of $200 \mathrm{~Hz}$. We therefore chose a $400 \mathrm{~Hz}$ digital cutoff frequency to reduce background noise while still preserving HCN kinetic information. See Kole et al. (2006) for an analysis of single HCN channel estimates versus filtering frequency.

The single-channel conductance $(\gamma)$ number of channels $(N)$ present were estimated by fitting a plot (Fig. $2 C, D$ ) of the ensemble variance versus the mean current (Fig. $2 B$ ) with Equation $8 . k$ represents the variance offset from underlying noise that is not time dependent, and was subtracted out in displayed plots (Fig. 2C,D).

$$
\sigma^{2}(I)=\gamma I-\frac{I^{2}}{N}+k
$$

\section{Results}

The rod and cone $I_{\mathrm{h}}$ currents are similar and dominated by

\section{HCN1 channels}

Whole-cell currents

Recordings of whole-cell currents were made from rods and cones in the whole-mount retina. We chose the whole-mount retinal preparation over the dissociated rod and cone preparations to avoid adverse effects associated with enzymatic or mechanical dissociation such as changes in channel kinetics/gating or loss of axon terminals. We found that contributions from adjacent rods to recorded currents via coupling were minimal, and did not affect our results (for explanation, see Materials and Methods, Recordings), as our data recorded from the intact retina also agree with experiments on dissociated salamander rods performed by us (Fig. 1A1) and Hestrin (1987). For whole-cell recordings, an extracellular solution containing TEA, cobalt, and barium was used to block all other ionic currents other than $I_{\mathrm{h}}$ (see Materials and Methods, Solutions). Although the whole-cell properties of the $I_{\mathrm{h}}$ current have been examined before in dissociated salamander photoreceptors or by using sharp electrodes (Hestrin, 1987; Maricq and Korenbrot, 1990; Akopian and Witkovsky, 1996; Malcolm et al., 2003), we felt the need to characterize them in our experiments using whole-cell voltage clamp in the intact retina, and standardize the recording conditions between rods and cones. This standardization allows us to directly compare our rod and cone data, compare results obtained from 
dark- and light-adapted conditions, and use our single-channel current data to estimate the number of channels present in each cell.

Our results show that the kinetic properties of $I_{\mathrm{h}}$ in whole-cell recordings of rods and cones are very similar, which suggests that the same channels carry $I_{\mathrm{h}}$ in both cell types. We also found that physiological properties of $I_{\mathrm{h}}$ in rods and cones in dark-adapted conditions are indistinguishable from those in light-adapted conditions, suggesting that $I_{\mathrm{h}}$ channels are not modulated by steady background light. Because HCN channels are also gated by cyclic nucleotides in addition to hyperpolarizing voltages, we attempted to measure the sensitivity of $\operatorname{rod} I_{\mathrm{h}}$ to cAMP. Although other researchers have reported a small modulatory effect of cAMP on the $I_{\mathrm{h}}$ activation curve in rabbit rods (Demontis et al., 2002), we were unable to produce a change in activation curve or $I-V$ curve with bath perfusion of 8 -Br-cAMP. This is consistent with reported evidence that $\mathrm{HCN} 1$ channels, which we believe are the primary isoform, are the least sensitive of the four HCN isoforms (Baruscotti et al., 2005). The steady state $I-V$ curves for rods and cones, generated by plotting the end steady state current at each holding potential, are both seen to be a hyperpolarization activated inward current with an amplitude of around $-150 \mathrm{pA}$ at $-134 \mathrm{mV}$ (Fig. 1A2,B2). This similarity in magnitude and rectification provides evidence that rods and cones have similar numbers of channels carrying the $I_{\mathrm{h}}$ current, assuming the identity of the channels is the same.

The steady-state activation curves, shown in Figure 1, A3 and $B 3$, generated by plotting the normalized amplitude of the tail currents at $-4 \mathrm{mV}$ versus the command pulse amplitude, are also similar. The half activation potential for rods, $-77.8 \pm 3.7 \mathrm{mV}$ (mean $\pm \mathrm{SD}$ ), is slightly more negative than for cones, $-73.8 \pm$ $1.2 \mathrm{mV}$. The activation rate constants are also similar (10.5 \pm 3.42 $\mathrm{mV}$ for rods and $7.1 \pm 0.86 \mathrm{mV}$ for cones), although the cone activation is slightly steeper. These activation curves for both cell types agree well with results published previously for rods (Hestrin, 1987; Maricq and Korenbrot, 1990; Demontis et al., 2002; Malcolm et al., 2003). Also similar are the rod and cone activation time constants (Fig. 1A4,B4), which were generated by fitting a single exponential decay to the traces in Figure $1, A 1$ and $B 1$. The similarity in the activation curves and time constants in rods and cones provides strong evidence that the kinetics, and therefore identity of the channels that carry rod and cone $I_{\mathrm{h}}$ is the same.

Most striking, however, is how closely the activation curves and activation time constants for rod and cone $I_{\mathrm{h}}$ resemble the activation curve and time constants for the HCN1 channel isoform. Colored lines in Figure 1, A3 and B3, show the activation curves for an allosteric model of HCN1, HCN2, and HCN4 channels. These allosteric models were developed from voltage-clamp data of homogenous populations of $\mathrm{HCN}$ isoforms expressed in human embryonic kidney cells (Altomare et al., 2001). HCN3 kinetics are known to be intermediate between HCN2 and HCN4 (Baruscotti et al., 2005). We estimated the parameters for HCN3 channels from another set of data (Stieber et al., 2005). The activation curves for rod and cone $I_{\mathrm{h}}$ appear to be similar to HCN1, $\mathrm{HCN} 3$, and HCN4 channels (Fig. 1A3,B3), however, the voltage dependence of the time constants closely resembles the time constants for HCN1 channels, and is significantly different from HCN2, HCN3, and HCN4 (Fig. 1A4,B4). The similarity in the activation curves and activation time constants for rod and cone $I_{\mathrm{h}}$ to the $\mathrm{HCN} 1$ isoform provides electrophysiological evidence that HCN channels carry the $I_{\mathrm{h}}$ current, and that HCN1 is the predominant $\mathrm{HCN}$ isoform present in both rods and cones.
Immunohistochemistry and Western blot

We used antibodies against the two known neuronal $\mathrm{HCN}$ isoforms (HCN1 and $\mathrm{HCN} 3$ ) to label $\mathrm{HCN}$ channels in the salamander retina. See methods for a description of the antibodies. Immunohistochemical staining of the salamander retina demonstrates stronger staining for $\mathrm{HCN} 1$ antibodies than $\mathrm{HCN} 3$ for rod and cone photoreceptors (Fig. 2). Anti-HCN1 antibodies strongly label the rod and cone inner segments, with some binding in the outer segments as well. This finding agrees with our electrophysiology data that indicate that the HCN1 isoform is dominant in these cells, however, we did not observe any HCN currents from the outer segments. HCN3 binding appears to be relatively nonspecific, with some binding in the photoreceptors.

To test the specificity of the antibodies used, a Western blot was performed on salamander retina and mouse brain. For HCN1 in mouse brain (Fig. 2A1), a single band appears in the $100-110 \mathrm{kDa}$ range, which is consistent with the predicted molecular weight for mouse HCN1 (102 kDa). For salamander, a single band appears near $60 \mathrm{kDa}$ (Fig. 2A1), which is consistent with findings of other researchers from the rat retina (Müller et al., 2003). Although the observed molecular weight is different from the mouse, the appearance of single specific band indicates that the HCN1 antibody does indicate specific binding in the salamander. A BLASTp search on the Xenopus tropicalis HCN1 protein sequence (Bowes et al., 2008) predicts binding of the HCN1 antibody to amphibian HCN1, with homology in 15 of 19 aa between the antibody epitope and Xenopus HCN1. The $60 \mathrm{kDa}$ band in retina has been observed by other researchers also using $\mathrm{N}$-terminal HCN1 antibodies in rat retina (Müller et al., 2003). It is possible that the $60 \mathrm{kDa}$ protein is a truncated form of $\mathrm{HCN} 1$. Western blot with the HCN3 antibody (Fig. 2) demonstrated distinct bands near $50 \mathrm{kDa}$ in both mouse brain and salamander retina. These bands may both represent protein fragments, as the predicted mass for mouse $\mathrm{HCN} 3$ is $85 \mathrm{kDa}$. Our results using immunohistochemical labeling and Western blot of whole retina, while they cannot rule out the presence of other $\mathrm{HCN}$ isoforms, are supportive of our electrophysiological findings that $\mathrm{HCN} 1$ is the dominant isoform present in photoreceptors.

\section{HCN1 single-channel conductance}

With evidence from whole-cell currents and immunohistochemical experiments indicating that the identity of the $I_{\mathrm{h}}$ current in rods and cones is the HCN1 channel, we endeavored to estimate the conductance of single HCN1 channels in the whole salamander retina. Normally the conductance of single ion channels is determined by directly observing single-channel events with a cell-attached patch, but the conductance of HCN channels is so small that it is below the thermal noise threshold of any physically realizable patch-clamp amplifier. Therefore we used a statistical technique called NSFA to estimate the conductance of single HCN channels in vivo (Sigworth, 1980; Alvarez et al., 2002; Hartveit and Veruki, 2007). An ensemble of 100 traces was recorded from whole-cell currents from rods using 100 traces of a command pulse to $-104 \mathrm{mV}$ (as in Fig. 3A), with other channel activity blocked with special external solutions (see Solutions in Materials and Methods). The variance and mean of the ensemble were then computed (as in Fig. 3B) (see Materials and Methods for detailed explanation) and plotted against one another (Fig. $3 C 2$ ). We initially used a whole-cell patch as opposed to the more common cell-attached technique to maximize the $I_{\mathrm{h}}$ current recorded. With this whole-cell NSFA recording technique, we estimated the HCN channel conductance to be $766 \pm 242 \mathrm{fS}$, with $2214 \pm 986(\mathrm{SD}, n=6)$ channels per rod (Fig. 3C2). 
A small activation delay, which is sometimes seen as an early minimum in HCN currents, is visible in Figures 2 and 3, $A$ and $B$. It appears to be more evident in recordings of cell-attached HCN currents. This activation delay has been observed in HCN currents recorded from Purkinje fibers (DiFrancesco, 1984), salamander rods (Hestrin, 1987), and expressed HCN channels (Männikkö et al., 2005). One explanation for this delay is that HCN channels have many different conformational states, and must transition to a lower conducting state shortly after a voltage pulse before reaching full activation.

While a larger $I_{\mathrm{h}}$ current can be recorded from a whole cell as opposed to a cell-attached patch, the cell-attached technique of recording currents has several advantages, including less membrane noise, avoidance of cell internal dialysis, and the ability to localize the distribution of $\mathrm{HCN}$ currents on the cell membrane (see Materials and Methods). Therefore, to confirm our whole-cell NSFA results, we also estimated the single-channel conductance of HCN channels with cell-attached patches, using special pipette solutions to increase the HCN driving force (see Materials and Methods, Solutions). The increased extracellular potassium level raised the reversal potential of HCN channels without affecting the gating kinetics of $I_{\mathrm{h}}$ (Wollmuth and Hille, 1992), increasing the driving force of $I_{\mathrm{h}}$ currents. These cell-attached patches in rods gave estimates of a single-channel conductance of $663 \pm 71 \mathrm{fS}$, with $155 \pm 42$

$(\mathrm{SD}, n=3$ ) channels present in each patch (Fig. $3 C 1)$. From the single-channel conductance we estimate the number of channels per cell by dividing the whole-cell conductance $(\mu=1.4 \pm 0.89$ $\mathrm{nS}$ ) by the single-channel conductance, which predicts $2111 \pm$ 1342 channels per cell. This result is similar to the number predicted with whole-cell NSFA $(N=2214)$. Due to the small size and the geometry of cones, long-term stable recordings (necessary for NSFA) of cone HCN currents were very challenging to obtain. However, recorded traces from cones gave an estimate of $526 \mathrm{fS}$, with 183 channels present per patch (Fig. 3D), which is similar to the single-channel conductance in rods. From this and the cone whole-cell conductance $(1.34 \pm 0.48 \mathrm{nS})$, we estimate there are $2021 \pm 725$ channels per cone. The comparable conductance and number of HCN channels in rods and cones is not surprising, because from analysis of the whole-cell currents, we show the identity of the channels is the same, and the magnitude of the whole-cell currents are similar (Fig. 1).

Our estimates of photoreceptor HCN conductance are comparable to the conductance reported for single $\mathrm{HCN}$ channels in rat cortical pyramidal neurons (Kole et al., 2006), which were also believed to be the HCN1 isoform. The significance of the similarity in conductance of HCN channels in photoreceptors and in pyramidal cells is twofold. First, this supports our whole-cell and immunohistochemical evidence that the HCN1 isoform is the dominant isoform expressed in photoreceptors. Second, it indicates that the conductance of HCN1 channels is similar in differ-
A

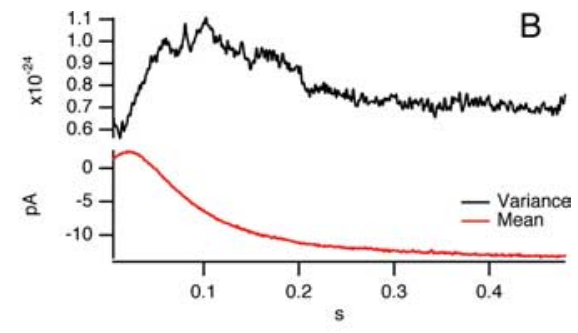

C1

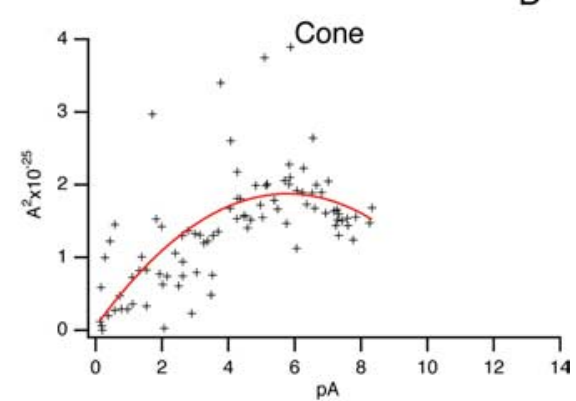

Figure 3. Nonstationary fluctuation analysis of $\mathrm{HCN}$ currents. $\boldsymbol{A}, \boldsymbol{B}$, Five traces from an ensemble $(\boldsymbol{A})$ and variance and mean channels in the patch (SD, $n=3$, two cells). $\mathbf{2}$, Whole-cell patches estimated the conductance to be $766 \pm 242 \mathrm{fS}$, with $N=$ $2214 \pm 986$ channels (SD, $n=6$ ). $\boldsymbol{D}$, In the cone, cell-attached patches yielded an estimated conductance of $526 \mathrm{fS}$ with 183 channels $(n=1)$ present per patch. Estimates were made from the coefficients of Equation 8.

ent species, which is consistent with genetic evidence showing that HCN channels are highly conserved across species (Jackson et al., 2007).

Comparison of the whole-cell and cell-attached NSFA allows us to make estimates of HCN channel density and distribution. HCN currents could only be observed with cellattached patches from the inner segment and cell bodies of rod and cone photoreceptors. This distribution is consistent with the proposed role of HCN channels as modulating the photocurrent's electrical signal as it propagates from the outer segment to the synapse. On-cell patches typically showed 1/14 as many channels as the whole cell. We estimate that the average surface area of rod and cone inner segments are 586 and $508 \mu \mathrm{m}^{2}$, respectively, from analysis of salamander retinal tissue sections (Zhang and $\mathrm{Wu}, 2009$ ). Assuming the channel density is even throughout the inner segment, this would correspond to a density of 3.6 channels $/ \mu \mathrm{m}^{2}$ in rods, and 4.0 channels $/ \mu \mathrm{m}^{2}$ in cones. These estimates of HCN channel density in the inner segment of rods and cones are similar to results reported by cell-attached currents recorded from areas near the soma of rat cortical neurons (Kole et al., 2006). Kole et al. (2006) report that HCN channel varies exponentially from 9 to 550 channels $/ \mu \mathrm{m}^{2}$ progressing from the soma to the distal end of the dendrites, an effect that had been previously noted in hippocampal pyramidal neurons (Magee, 1998). 

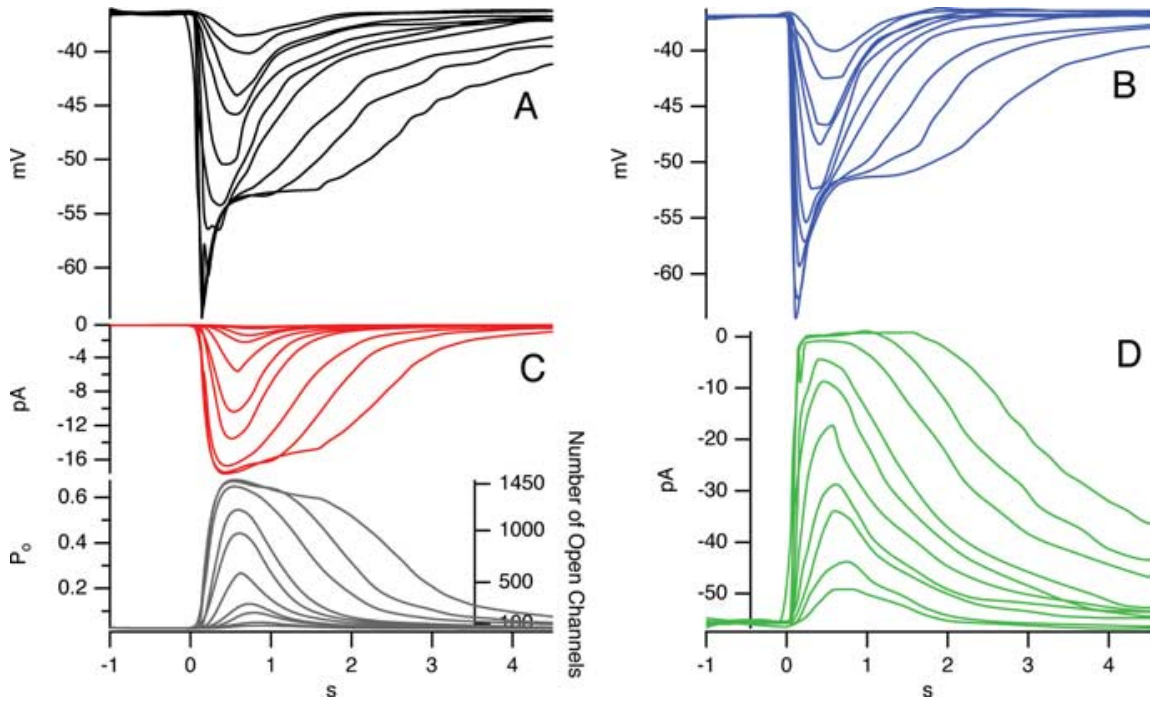

Figure 4. Simulation analysis of rod photoreceptor function. $A$, Simulated voltage response of a rod to increasing light intensities. $\boldsymbol{B}$, Actual voltage response recorded simultaneously with the photocurrent in $\boldsymbol{D}$ from an isolated rod. Data for $\boldsymbol{B}$ and $\boldsymbol{D}$ are taken from Baylor and Nunn (1986). $C, I_{\mathrm{h}}$ current and opening probability $P_{0}$ with corresponding number of open $\mathrm{HCN}$ channels for each stimulus as predicted by simulation.
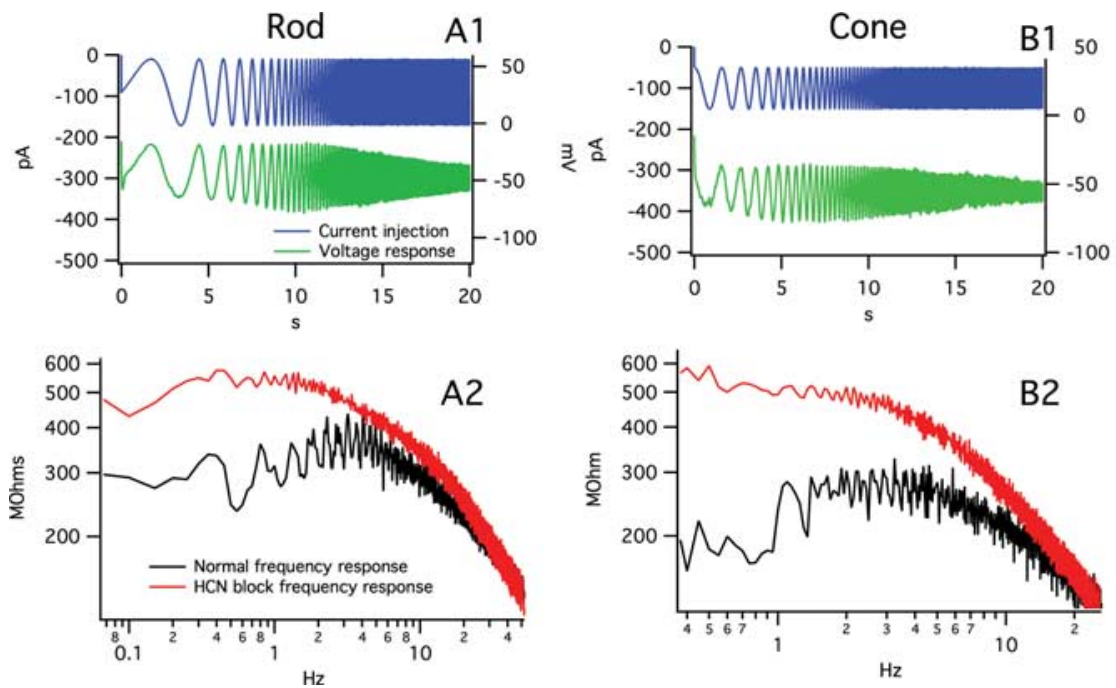

Figure 5. $A \mathbf{A} 1, \boldsymbol{B} 1$, Stimulus and response of a $\operatorname{rod}(\boldsymbol{A} 1)$ and cone $(\boldsymbol{B} 1)$ to a frequency-chirped sine wave current stimulus. $\boldsymbol{A} \mathbf{2}, \boldsymbol{B} \mathbf{2}$, Rod $(\boldsymbol{A Z})$ and cone $(\boldsymbol{B} 2)$ frequency response determined from stimulus and response, plotted on log-log axes. Application of ZD 7288 , which blocks HCN channels, is shown in red. Normal rods and cones are seen to have a peak frequency response near $4 \mathrm{~Hz}$. HCN channel block is seen to abolish the bandpass filter characteristic, leaving a low-pass filter resulting from the membrane capacitance and resistance.

\section{Contribution of single $\mathrm{HCN}$ channels to the rod light response}

To determine HCN1 channel contributions to light evoked responses in photoreceptors, we simulated the light response of a rod photoreceptor, incorporating the data from our singlechannel conductance and whole-cell studies of HCN1 channels (described above) into the simulation (Fig. 4A). The numerical simulation model was developed with photocurrent data from the suction electrode technique experiments of Baylor and Nunn (1986), and our HCN channel data combined with models of other voltage-gated currents, as developed by other salamander rod photoreceptor models (Kamiyama et al., 1996; Ogura et al., 2003; Liu and Kourennyi, 2004; Publio et al., 2006). The simulation approximates the voltage response of an actual rod photore- ceptor at multiple light intensities (Fig. $4 A, B)$. By using actual data for the photocurrent (Fig. 4D) and comparing the model response to the actual voltage response, we made every effort to make our model reflect not only the voltage response of a photoreceptor, but the contribution of individual ionic currents. Through the simulation, we were able to predict the time course and magnitude of the $I_{\mathrm{h}}$ current during the voltage response of a rod. Using our data for the single-channel conductance, we were also able to estimate the number of HCN channels open at any given instant in time during a typical flash light response. The model predicts an opening probability $P_{\mathrm{o}}=0.02$ at the dark membrane potential, which corresponds to an average of $\sim 44$ open HCN channels. This means that in darkness and at very dim light intensities, only a few $\mathrm{HCN}$ channels contribute to the resting potential. On the other hand, in response to a bright flash, HCN channels reach a peak open probability of $P_{\mathrm{o}}=0.65$, which corresponds to 1430 open $\mathrm{HCN}$ channels (Fig. 4C). The model also shows that once ₹ they are opened by the fast initial hyperpolarization during the light response, $\mathrm{HCN}$ channels are slow to close after the sharp "nose" in the voltage response. This finding uncovers a potential advantage for the extremely low single-channel conductance observed by our NSFA studies in photoreceptors. Namely, at a low opening probability, as in darkness and dim light, greater numbers of low-conductance channels spontaneously opening and closing (as found with photoreceptor $I_{\mathrm{h}}$ ) would cause less noisy fluctuations in the membrane potential than small numbers of highconductance channels. This means that a low single HCN channel conductance could help improve the signal-to-noise ratio of rods in dim light.

\section{HCN contribution to photoreceptor intrinsic frequency response}

Frequency-chirped sinusoidal current stimuli according to Equation 1 were injected into both rods and cones to examine the contribution of $\mathrm{HCN}$ channels to the electrical frequency response of these cells. All other currents were blocked (see Materials and Methods, Solutions), and the voltage responses were recorded (Fig. 5A1,B1). The magnitude of the Fourier transform of the stimulus and response were computed, and the impedance was plotted as $Z(f)=|V(f)| /|I(f)|$. This analysis demonstrated that HCN channels cause a peak in the electrical impedance of both rod and cone photoreceptors at $\sim 4$ $\mathrm{Hz}$, an effect equivalent to a bandpass filter (Fig. 5A2,B2, black traces).

Application of $100 \mu \mathrm{M}$ ZD 7288, which selectively blocks HCN channels (Satoh and Yamada, 2000; Kawai et al., 2005), was found to abolish the bandpass filter response (Fig. 5A2,B2, red 
traces). The frequency responses with HCN channels blocked with ZD7288 are those of a passive membrane, and equivalent to a low-pass filter. These results reveal that the bandpass filter effect seen when HCN channels are active arises because of a combination of an $I_{\mathrm{h}}$-mediated high-pass filter, and a low-pass filter formed from the membrane resistance and capacitance. In other words, HCN channels serve to lower the membrane impedance at low frequencies, which means the cell will be less sensitive to inputs at these frequencies.

From a theoretical perspective, a passive rod network would be modeled electrically with cells represented by the parallel combination of a resistor and capacitor, coupled to a neighboring cell of the same configuration by a resistor. This is the same as the cable equation. Because the network has only parallel resistances and capacitances and no series capacitance, it can act only as a low-pass filter. Changes in passive membrane resistance by blocking $I_{\mathrm{h}}$ can only cause a shift in the cutoff frequency of the low-pass filter, and not the high-pass filter characteristic observed in these experiments.

From an experimental standpoint, although our chirped current injection experiments were performed in the wholemount retina, we were able to observe the same bandpass effect in cone photoreceptors as in rods. Salamander cones have been shown to have much weaker coupling to adjacent rods than rod-rod coupling, which would mean that if the bandpass filter effect were from the network, the effect should be much less for cones than rods. Because we instead observe a very similar bandpass filtering effect in cones and rods, it is likely that the effect comes from the electrical properties of HCN channels in individual cells, than from the coupled network. We believe that the bandpass filtering observed in this and other studies (Zhang and $\mathrm{Wu}, 2005)$ is a result of active voltage-gated conductances, such as $I_{\mathrm{h}}$, and not the coupled rod network.

\section{Contribution to step response and equivalent circuit}

With the realization that the electrical characteristics of the cell are equivalent to the combination of an HCN-mediated highpass filter and a low-pass filter from the cell membrane, an equivalent circuit can be constructed (Fig. 6C). The voltage response of a rod and cone due to the $I_{\mathrm{h}}$ current at given input current can be modeled as a linear time-dependent circuit (Detwiler et al., 1978, 1980; Owen and Torre, 1983; Torre and Owen, 1983). This circuit model has been studied before, and demonstrates what is referred to as the "inductive" property of the photoreceptors due to $I_{\mathrm{h}}$ (Detwiler et al., 1978, 1980; Owen and Torre, 1983; Torre and Owen, 1983; Demontis et al., 1999). We have extended previous work by using this model to create a linear approximation of the photoreceptor electrical response at different input magnitudes, and use this model predict the change in frequency response of the cell with stimulus intensity.

In the circuit, the capacitor $C$ represents the membrane capac- itance, $R_{\mathrm{m}}$ the membrane resistance, and the inductor $L$ and series resistance $R_{1}$ the contribution of $I_{\mathrm{h}}$. The circuit works as follows: injected current charges the membrane capacitance with a time constant dependent on $R_{\mathrm{m}}$ and $C$. After a delay, the inductor $L$ representing the contribution of HCN channels begins to turn on and shunt current through its branch of the circuit with a time constant dependent on $L$ and $R_{1}$. This causes a sag in the voltage response, shown in Figure 6, $A$ and $B$.

To determine the values for the equivalent circuit, hyperpolarizing current pulses were delivered to rods and cones, and the voltage responses were recorded (Fig. 6A,B). From these responses the parameters for each component of the circuit were estimated, and the corresponding frequency response predicted. The membrane capacitance and whole-cell resistance were determined by fitting the first hyperpolarizing pulses that failed to activate $I_{\mathrm{h}}$. Parameters for $L$ and $R_{1}$ at each input level were determined using a least-squares fit for the equivalent circuit voltage response. This model shows that as the hyperpolarizing input magnitude increases, the inductance $L$ and series resistance $R_{1}$ in the equivalent circuit decrease in both rods and cones (Fig. $6 A 3, A 4, B 3, B 4)$. This causes a corresponding increase in the resonant frequency and damping factor (Fig. 6A1,A2,B1,B2). Plotting the model predicted frequency response demonstrates the shift in peak response for rods and cones with increasing stimulus strength (Fig. $7 A, B$ ). These shifts, from $\sim 2$ to $4 \mathrm{~Hz}$ for rods, and from 1.5 to $3.5 \mathrm{~Hz}$ for cones, suggest that the HCN channels work to extend the operational frequency range for both rod and cone 

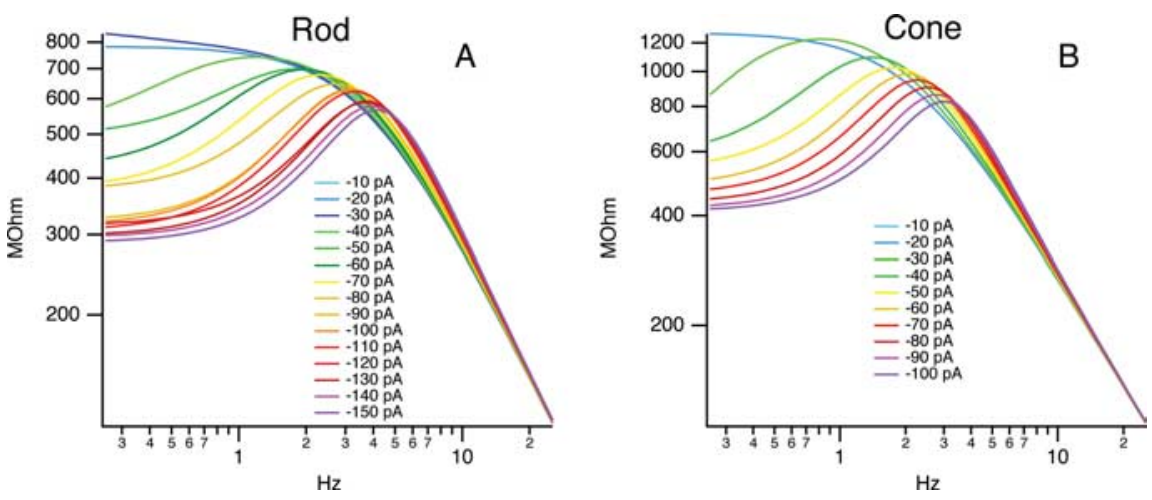

Figure 7. Model (Fig. 6)-predicted frequency response to electrical stimuli. $\boldsymbol{A}$, Frequency response of a rod photoreceptor predicted by the inductive model for each input magnitude. $\boldsymbol{B}$, Frequency response of cone photoreceptor. This demonstrates the $\mathrm{HCN}$-mediated bandpass filter response of rods and cones at different input magnitudes. The resonant frequency shifts from $1 \mathrm{~Hz}$ to $\sim 4 \mathrm{~Hz}$ for both rods and cones.
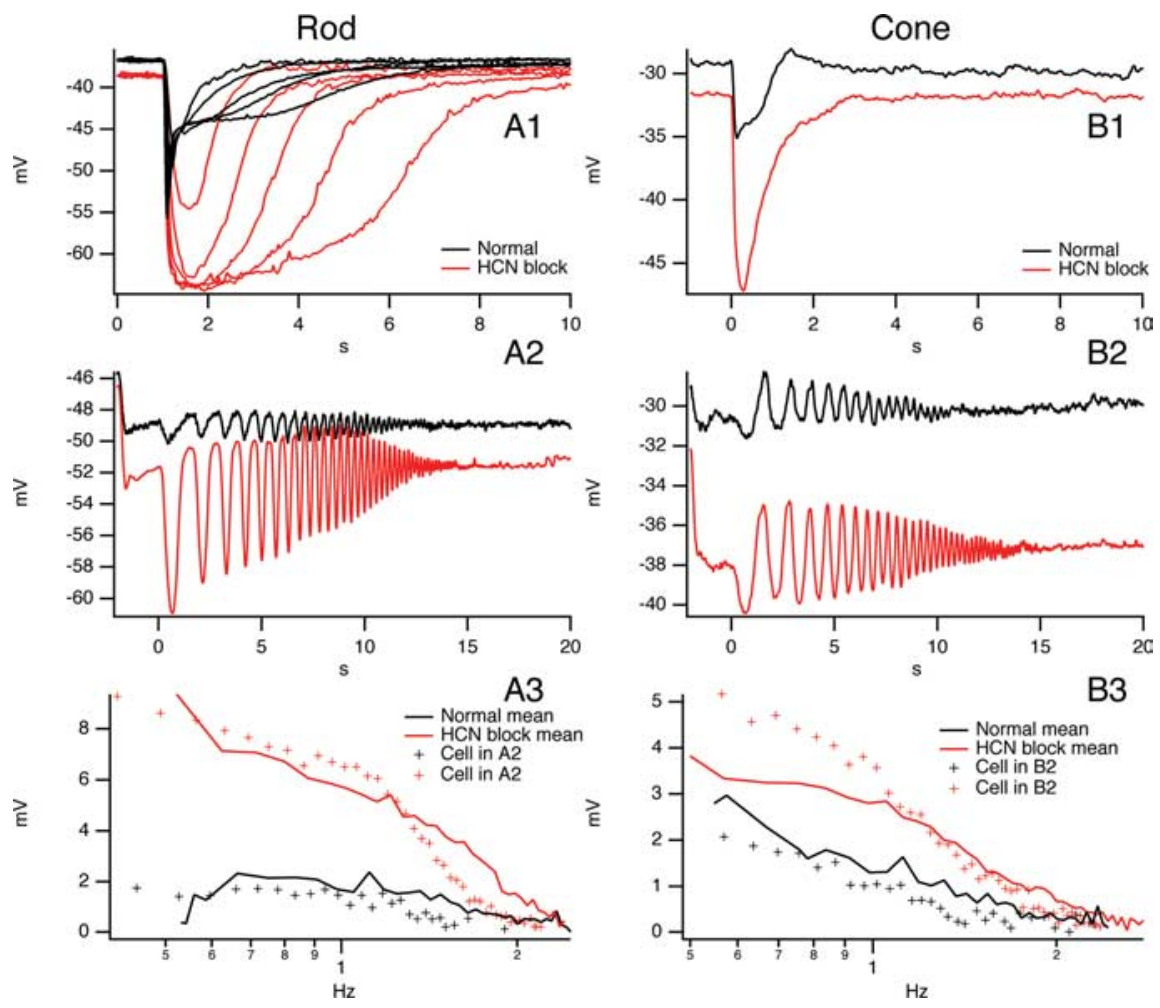

Figure 8. Rod light responses. $\boldsymbol{A}$, Normal rod light response to flashes of increasing light intensity. $\boldsymbol{B} 1$, Normal cone response to a flash of light. Response to the same stimulus after blocking HCN channels with ZD 7288 is shown in red. $\mathbf{A 2}, \mathbf{B 2}, \operatorname{Rod}(\boldsymbol{A 2})$ and cone (B2) response to frequency-chirped light stimulus before and after $\mathrm{HCN}$ block. $\boldsymbol{A} \mathbf{3}$ and $\boldsymbol{B} \mathbf{3}$ demonstrate the amplitude versus frequency for chirped light stimuli. Solid lines show population means ( $n=4$ for $A \mathbf{3}$ and $n=3$ for $B 3$ ), while crosses show the data for traces shown in $\mathbf{A} \mathbf{2}$ and $\mathbf{B} \mathbf{2}$, respectively. When HCN channels are blocked, there is a steady decay in the frequency response of both rod and cone photoreceptors, at dim and bright light intensities, respectively.

photoreceptors. This effect, along with the bandpass filter response, helps to quicken the voltage response of photoreceptors.

\section{How HCN channels shape responses to rod and cone photocurrents}

Up to this point we have shown that the single-channel conductance, whole-cell current, and electrical characteristics of the $I_{\mathrm{h}}$ current are very similar in rods and cones. However, results using light stimuli demonstrate that the effect of $\mathrm{HCN}$ channels on the light response of rods and cones differs in accordance with the distinct function of these two cell types.
Flash response

Applying $50 \mu \mathrm{M}$ of the HCN blocker ZD 7288 greatly increased the amplitude of the light response to a bright flash for both dark adapted rods and cones (Fig. $8 A 1, B 1)$. In rods, blocking $\mathrm{HCN}$ channels abolished the transient "nose" present in the light response (Fig. 8A1), while in cones, blocking it increased the response magnitude and appeared to eliminate the voltage overshoot present in the recovery phase (Fig. 7B1). With $\mathrm{HCN}$ channels blocked, the light response magnitude and time course for rods and cones are both increased for flash stimuli. This means that $\mathrm{HCN}$ channels play a role in aiding recovery of from bright stimuli in both cell types from a dark-adapted baseline.

\section{Chirped light stimuli}

To measure the contribution of $\mathrm{HCN}$ channels to the frequency response of photoreceptors in response to light, their natural stimulus, chirped sine wavemodulated light stimuli were generated that ranged from 0.5 to $5 \mathrm{~Hz}$ for a rod over the course of $20 \mathrm{~s}$ (Eq. 1). These stimuli were similar to the electrical stimuli described previously, but in the form of light rather than current. Background illumination for $2 \mathrm{~s}$ adapted the retina to the average light intensity of the stimulus. In a normal rod the light stimulus produced a response of consistent amplitude from 0.5 to $1 \mathrm{~Hz}$ at $1 \times 10^{-2}$ lux (Fig. $8 \mathrm{~A} 2$ ), falling off rapidly at higher frequencies (Fig. 8 A3). However, when $\mathrm{HCN}$ channels were blocked with ZD 7288, the response was much greater in magnitude, especially at low frequencies (Fig. 8A2, red trace). The frequency components of the $\mathrm{HCN}$ blocked response were seen to decay beginning at $0.5 \mathrm{~Hz}$, similar to a low-pass filter (Fig. 8A3). The decline at frequencies $>1 \mathrm{~Hz}$ was much more rapid than the falloff of the low-pass filter of the electrical stimuli (Fig. 7), and had a lower cutoff frequency. This suggests that the components of this decay are due to the limited frequency response of the photocurrent, and not the low-pass filter of the cell membrane. Cones saw a similar increase in lowfrequency components with HCN channels blocked, although the light intensities to achieve this affect were greater $\left(5.46 \times 10^{-2} \mathrm{lux}\right)$. The voltage falloff at $1 \mathrm{~Hz}$ was less sharp than in rods, which is consistent with the faster kinetics of the cone photocurrent. Both rods and cones showed an increase in low-frequency amplitudes, and greater frequency-dependent decay with HCN block. Comparing the normal light frequency responses to those with HCN channels blocked demonstrates that HCN channels act as a compensator that cancels out the frequency-dependent decay in the light response over the range from 0 to $1 \mathrm{~Hz}$. On a cellular level, slowly changing hyperpolar- 
izing stimuli turn on HCN channels, which turn on and shunt the original stimulus, reducing its intensity.

Light impulse response

The impulse responses of rod and cone photoreceptors were estimated using GWN light stimuli according to the Lee-Schetzen method (Marmarelis and Marmarelis, 1978). A GWN stimulus approximates physiologic conditions for photoreceptors, with luminance in a visual scene fluctuating around a steady mean, as opposed to a flash stimulus, which measures the dark-adapted response to a single flash impulse. Briefly, the impulse response demonstrates the duration of time necessary for a system to respond to an infinitely brief stimulus. At dim light intensities $\left(1.19 \times 10^{-2}\right.$ lux $)$, the impulse response of a normal rod is shown to increase in magnitude and duration when HCN channels are blocked with ZD 7288 (Fig. 9A1). However, at brighter light intensities, the impulse response was relatively unchanged by blocking HCN channels (Fig. 9A2). By shortening the duration of the impulse response, HCN channels reduce the amount of time necessary for rods to encode an impulse of information in dim conditions. The lack of effect at brighter intensities could be due to saturation of the rod light response. At a higher mean luminance level, impulses of light or darkness on top of the mean luminance are not able to cause significant changes in the rod voltage response, and as a result, these smaller fluctuations do not cause significant changes in HCN channel activation. Figure 8 shows that HCN blockade does not affect the light impulse response much at brighter mean luminances. HCN channels appear to be most effective for the normal operating state of rod photoreceptors - under dim light.

On the other hand, in cones, the impulse response to dim light $\left(2.46 \times 10^{-2}\right.$ lux $)$ was seen to be relatively unaffected by blocking HCN channels (Fig. 9B1). This is likely due to minimal HCN activation by cones at dim light intensities. At brighter light intensities $\left(5.46 \times 10^{-2} \mathrm{lux}\right), \mathrm{HCN}$ block caused an increase in the magnitude and duration of the impulse response, similar to the change seen in rods at low light intensities (Fig. 9B2). This implies that under brighter light, the normal operating condition for cones, HCN channels are functioning optimally, and help to reduce the amount of time needed to encode an impulse of information. The differential effect of dim versus bright light on the function of HCN channels in rod and cone photoreceptors coincides with the normal operating characteristic of these two cell types. Rods, which are sensitive to small changes in light intensity in dim light, see the optimal contribution of HCN channels in these conditions, whereas cones, which are sensitive to brighter light, see the optimal effect of HCN channels at brighter light intensities.

The Fourier transform of the impulse response gives the estimated frequency response from the GWN stimulus. This is shown for the rod and cone where HCN channels are effec- tive-at dim and bright light intensities, respectively (Fig. $9 A 3, B 3)$. These estimates of the rod and cone light-mediated frequency responses are similar to our estimates using chirped light stimuli (Fig. 8A3,B3), with a frequency-dependent decay at low frequencies in both rods and cones with HCN block. The similarity in the frequency responses predicted from the GWN technique and those from the chirped light stimuli confirms that photoreceptors have a mostly linear response component (Marmarelis and Naka, 1973), and supports our use of GWN estimated impulse responses to approximate rod and cone function. It also shows how a the changes in the rod and cone impulse response functions with $\mathrm{HCN}$ block correspond to the low-pass roll off characteristic seen with the frequency-chirped light stimuli.

\section{Discussion}

$I_{\mathrm{h}}$ in salamander rods and cones is mediated by HCN1 channels

In this study, we systematically investigate the biophysical and physiological properties of HCN channels in salamander rod and cone photoreceptors. We show that the $I_{\mathrm{h}}$ current in rod and cone photoreceptors is alike with respect to the whole-cell current magnitude, activation curve and time course, and that these values are characteristic of the HCN1 isoform (Fig. 1 A1-A4,B1$B 4)$. Our whole-cell data from salamander rods are similar to reports from other researchers (Hestrin, 1987; Maricq and Korenbrot, 1990; Akopian and Witkovsky, 1996; Malcolm et al., 
Table 1. Kinetic parameters for $I_{h}$ and HCN isoforms

\begin{tabular}{lcc}
\hline HCN isoform & $V_{1 / 2} \pm S D(m V)$ & $t \pm S D$ at $-94 \mathrm{mV}(\mathrm{ms})$ \\
\hline Rod $I_{\mathrm{h}}$ & $-77 \pm 0.78(n=10)$ & $52.3 \pm 9.5(n=4)$ \\
Cone $I_{\mathrm{h}}$ & $-74 \pm 1.1(n=10)$ & $50.7 \pm 8.0(n=8)$ \\
HCN1 & -69 & 84 \\
HCN2 & -95 & 557 \\
HCN3 & -77 & 1408 \\
HCN4 & -81 & 3586
\end{tabular}

Values for rod and cone $I_{\mathrm{h}}$ are very similar. Kinetic data for both rods and cones are similar to HCN1. Data for HCN1-HCN4 come from measurements from homogeneous expression systems (Altomare et al., 2001; Stieber et al., 2005). The half-activation voltage for $\mathrm{HCN} 1$ is known to be $\sim 5 \mathrm{mV}$ more hyperpolarized in in vivo systems than in the expression system (Altomare et al., 2001).

2003), however, we use the intact retina to avoid the drawbacks of dissociated cells and standardize recording conditions for rods and cones. With whole-cell voltage clamp, we demonstrate that the activation time course of rod and cone $I_{\mathrm{h}}$ is similar to homogeneously expressed HCN1 channels (Fig. 1 A3,B3, Table 1). Although the rod and cone half activation potentials are more hyperpolarized than the expressed HCN1 channels, this is a known difference between in vivo versus expressed $\mathrm{HCN} 1$ channels (Altomare et al., 2003). While other researchers have shown that the HCN1 isoform is responsible for $I_{\mathrm{h}}$ in rabbit rods (Demontis et al., 2002), we demonstrate that this is true not only for salamander rods, but also for cones. Our immunohistochemical and Western blot experiments support our finding that HCN1 channels are responsible for the $I_{\mathrm{h}}$ current in rods and cones (Fig. 2 ). These results, which agree with experiments on the rat retina (Müller et al., 2003), show anti-HCN1 antibodies strongly labeling rods and cones. Our Western blot experiments show that the $\mathrm{HCN} 1$ antibody binds to a $60 \mathrm{kDa}$ protein, which was also noted by Müller et al. (2003) in the rat retina. Given the specificity predicted by a BLASTp search (see Results and Materials and Methods) and demonstrated in the blot itself (Fig. 2A1), we believe that the $60 \mathrm{kDa}$ protein represents a truncated form of $\mathrm{HCN} 1$ in the retina. Whether this product is active or not warrants further investigation.

\section{Conductance of single $\mathrm{HCN} 1$ channels}

By using NSFA, we provide the first known estimate of a singlechannel conductance in the retina. We show that photoreceptor $\mathrm{HCN}$ channels have a conductance of $\sim 663 \mathrm{fS}$ (Fig. 3), comparable to HCN1 conductance in rat cortical neurons (Kole et al., 2006). This similarity is in line evidence demonstrating that HCN channels are highly conserved across species (Jackson et al., 2007). We find that HCN channels are expressed in the inner segments of rods and cones, with $\sim 2000$ channels per cell. The density of these channels in the salamander rod and cone inner segment appears to be similar to the density of HCN channels near the soma of rat pyramidal cells, but less than the density near the distal ends of dendrites (see Results) (Magee, 1998; Kole et al., 2006). Of the many channels present, only a small number are active at the rod dark membrane potential (Fig. 4). With only a few channels open in dim light, the extremely low single-channel conductance of HCN channels may be advantageous by minimizing membrane noise.

\section{HCN1 channels create a bandpass filter in photoreceptors}

To investigate how HCN channels affect the electrical properties of rods and cones, we examined the effect of $\mathrm{HCN}$ channels on their responses to extrinsic electrical stimuli. Using frequencychirped stimuli, we demonstrate that HCN channels turn the low-pass filter characteristic of the rod and cone membrane into a bandpass filter, with a peak of $\sim 4 \mathrm{~Hz}$ for both cells (Fig. 5). This is similar to observations of the frequency response in the dendrites of rat hippocampal neurons (Narayanan and Johnston, 2007). Although the bandpass filtering effect has been investigated before in rod photoreceptors (Detwiler et al., 1978, 1980; Attwell and Wilson, 1980; Owen and Torre, 1983; Torre and Owen, 1983; Baylor et al., 1984; Demontis et al., 1999; Zhang and $\mathrm{Wu}, 2005)$, we show that cones also exhibit a similar bandpass filter effect, and that the frequency response of rods and cones varies with input intensity. By injecting hyperpolarizing steps of current into rods and cones we analyze a circuit analog for photoreceptors (Fig. 6C), which predicts that the frequency response of the bandpass filter becomes more peaked and shifts to higher frequencies as stimulus intensity increases (Fig. $7 A, B$ ). These effects are observed within individual photoreceptors, and are independent of the surrounding network.

\section{HCN1 channels help rods and cones efficiently encode impulses of light}

To investigate how HCN channels shape the light response of rods and cones, we use pharmacology combined with various light stimuli. With chirped light stimuli, we show that HCN channels act as a compensator, or damper, that normalizes the frequency-dependent decay of the light response in both rods and cones (Fig. 8A3,B3). Using GWN light stimuli, we estimate the impulse responses of rods and cones at different mean luminances. We find that $\mathrm{HCN}$ channels reduce the amount of time needed for rods to respond to an impulse of information in conditions of low mean luminance (Fig. 9A1), but that this effect saturates at brighter light intensities (Fig. 9A2). Conversely, in cones, $\mathrm{HCN}$ channels reduce the time to respond to an impulse of information at brighter light intensities (Fig. 9B2), but have little effect at dim light intensities (Fig. 9B1). Previous work has done little to clearly explain the functional advantage of these channels other than describe the bandpass filtering effect $I_{\mathrm{h}}$ has on the rod network. Our analyses show that in acting as a high-pass filter, $\mathrm{HCN}$ channels reduce the time needed for rods and cones to respond to encode visual information in the optimal operating conditions for each cell type-dim light for rods, and brighter light for cones. One explanation for this effect is the difference in the photocurrents of each cell. Rods, which have a slow but high gain photocurrent, operate within the active voltage range for $\mathrm{HCN}$ channels at dim light intensities, but at brighter light intensities their photocurrent saturates, and is no longer able to be filtered by HCN channels. On the other hand, cones have a faster but lower gain photocurrent, which does not use the operational range of $\mathrm{HCN}$ channels unless the cell is stimulated with brighter light (Fig. 9). Therefore, although the biophysical and electrical properties of HCN channels are similar in rods and cones, the channels' effect on rod and cone light responses is specific to the distinct function of these photoreceptors.

Despite its importance in shaping the rod and cone light response, $I_{\mathrm{h}}$ is not the only ionic current that plays a role in bandpass filtering in rod photoreceptors. $I_{\mathrm{Kx}}$, a potassium current, also plays a role in shaping the rod light response (Beech and Barnes, 1989; Liu and Kourennyi, 2004), but its role in cones is still unclear (Barnes and Hille, 1989). $I_{\mathrm{Kx}}$, similar to $I_{\mathrm{h}}$, exerts a depolarizing force on the membrane potential when the membrane is hyperpolarized. Traditionally, $I_{\mathrm{Kx}}$ is thought to be the primary mediator of high-pass filtering of small signals in rods because it is active around the dark membrane potential, while $I_{\mathrm{h}}$ is involved with filtering larger signals (Beech and Barnes, 1989). Contrary to these beliefs, we show that although $I_{\mathrm{h}}$ is minimally active at the 
rod dark membrane potential, it still affects the rod light response in dim conditions (Fig. 9). Therefore, it appears that both $I_{\mathrm{h}}$ and $I_{\mathrm{Kx}}$ are important in accelerating dim light responses.

\section{Conclusion}

In the retina, HCN channels appear to be necessary for the retina's temporal response to light stimuli. Blocking them reduces its ability to respond to quickly changing light stimuli (Gargini et al., $1999 b, c)$. Although HCN expression is not limited to the photoreceptors (Müller et al., 2003), our results suggest that their role in photoreceptors can explain in part the loss of the retina's temporal resolution with HCN block. Changes in the rod and cone responses to flash, chirped, and GWN light stimuli with HCN block all show a significant slowing of the response kinetics. In light of these observations, it also makes sense that HCN1, the fastest of the four $\mathrm{HCN}$ isoforms, is dominant in these cells. Incidentally, we also find that HCN activation does not appear to be affected by background light, which is consistent with the very low cyclic nucleotide sensitivity reported for the HCN1 isoform (Baruscotti et al., 2005). In addition to elucidating how HCN channels function in photoreceptors, our study also sheds light on our understanding of the visual side effects of a new class of heart medications that target cardiac HCN channels to slow heart rate (Bucchi et al., 2006; Stieber et al., 2006). The visual side effects of these drugs come from their action on HCN channels in the retina, which may well be due to blocking $\mathrm{HCN}$ channels in photoreceptors. Our work, which shows that HCN1 is the dominant isoform in rod and cone photoreceptors, suggests that newer cardiac drugs may be able to minimize visual side effects by selectively blocking HCN2 and $\mathrm{HCN} 4$, the cardiac $\mathrm{HCN}$ isoforms (Bucchi et al., 2006). A newly developed HCN blocker, ivabradine, appears to exhibit some therapeutic properties in this direction, and may lead the way for a shift in treatment of patients with heart disease to more specific bradycardic agents (Fox et al., 2008a,b).

\section{References}

Akopian A, Witkovsky P (1996) D2 dopamine receptor-mediated inhibition of a hyperpolarization-activated current in rod photoreceptors. J Neurophysiol 76:1828-1835.

Altomare C, Bucchi A, Camatini E, Baruscotti M, Viscomi C, Moroni A, DiFrancesco D (2001) Integrated allosteric model of voltage gating of hcn channels. J Gen Physiol 117:519-532.

Altomare C, Terragni B, Brioschi C, Milanesi R, Pagliuca C, Viscomi C, Moroni A, Baruscotti M, DiFrancesco D (2003) Heteromeric hcn1-hcn4 channels: a comparison with native pacemaker channels from the rabbit sinoatrial node. J Physiol 549:347-359.

Alvarez O, Gonzalez C, Latorre R (2002) Counting channels: a tutorial guide on ion channel fluctuation analysis. Adv Physiol Educ 26:327-341.

Attwell D, Wilson M (1980) Behaviour of the rod network in the tiger salamander retina mediated by membrane properties of individual rods. J Physiol 309:287-315.

Barnes S, Hille B (1989) Ionic channels of the inner segment of tiger salamander cone photoreceptors. J Gen Physiol 94:719-743.

Baruscotti M, Bucchi A, Difrancesco D (2005) Physiology and pharmacology of the cardiac pacemaker ("funny") current. Pharmacol Ther 107:59-79.

Baylor DA, Nunn BJ (1986) Electrical properties of the light-sensitive conductance of rods of the salamander Ambystoma tigrinum. J Physiol 371:115-145.

Baylor DA, Matthews G, Nunn BJ (1984) Location and function of voltagesensitive conductances in retinal rods of the salamander, Ambystoma tigrinum. J Physiol 354:203-223.

Beech DJ, Barnes S (1989) Characterization of a voltage-gated K+ channel that accelerates the rod response to dim light. Neuron 3:573-581.

Bowes JB, Snyder KA, Segerdell E, Gibb R, Jarabek C, Noumen E, Pollet N,
Vize PD (2008) Xenbase: a Xenopus biology and genomics resource. Nucleic Acids Res 36:D761-7.

Bucchi A, Tognati A, Milanesi R, Baruscotti M, DiFrancesco D (2006) Properties of ivabradine-induced block of hen 1 and hen 4 pacemaker channels. J Physiol 572:335-346.

Demontis GC, Longoni B, Barcaro U, Cervetto L (1999) Properties and functional roles of hyperpolarization-gated currents in guinea-pig retinal rods. J Physiol 515:813-828.

Demontis GC, Moroni A, Gravante B, Altomare C, Longoni B, Cervetto L, DiFrancesco D (2002) Functional characterisation and subcellular localisation of hen 1 channels in rabbit retinal rod photoreceptors. J Physiol 542:89-97.

Detwiler PB, Hodgkin AL, McNaughton PA (1978) A surprising property of electrical spread in the network of rods in the turtle's retina. Nature 274:562-565.

Detwiler PB, Hodgkin AL, McNaughton PA (1980) Temporal and spatial characteristics of the voltage response of rods in the retina of the snapping turtle. J Physiol 300:213-250.

DiFrancesco D (1984) Characterization of the pace-maker current kinetics in calf Purkinje fibres. J Physiol 348:341-367.

DiFrancesco D (2006) Serious workings of the funny current. Prog Biophys Mol Biol 90:13-25.

Fesenko EE, Kolesnikov SS, Lyubarsky AL (1985) Induction by cyclic GMP of cationic conductance in plasma membrane of retinal rod outer segment. Nature 313:310-313.

Fox K, Ford I, Steg PG, Tendera M, Ferrari R, on behalf of the BEAUTIFUL investigators (2008a) Ivabradine for patients with stable coronary artery disease and left-ventricular systolic dysfunction (beautiful): a randomised, double-blind, placebo-controlled trial. Lancet 372:807-816.

Fox K, Ford I, Steg PG, Tendera M, Robertson M, Ferrari R, on behalf of the BEAUTIFUL investigators (2008b) Heart rate as a prognostic risk factor in patients with coronary artery disease and left-ventricular systolic dysfunction (beautiful): a subgroup analysis of a randomised controlled trial. Lancet 372:817-821.

Gargini C, Demontis GC, Cervetto L, Bisti S (1999a) Analysis of pharmacologically isolated components of the erg. Vision Res 39:1759-1766.

Gargini C, Demontis GC, Bisti S, Cervetto L (1999b) Effects of blocking the hyperpolarization-activated current (ih) on the cat electroretinogram. Vision Res 39:1767-1774.

Gargini C, Demontis GC, Bisti S, Cervetto L (1999c) Temporal fidelity in the visual system. Arch Ital Biol 137:299-309.

Hamill OP, Marty A, Neher E, Sakmann B, Sigworth FJ (1981) Improved patch-clamp techniques for high-resolution current recording from cells and cell-free membrane patches. Pflugers Arch 391:85-100.

Hartveit E, Veruki ML (2007) Studying properties of neurotransmitter receptors by non-stationary noise analysis of spontaneous postsynaptic currents and agonist-evoked responses in outside-out patches. Nat Protoc 2:434-448.

Hestrin S (1987) The properties and function of inward rectification in rod photoreceptors of the tiger salamander. J Physiol 390:319-333.

Jackson HA, Marshall CR, Accili EA (2007) Evolution and structural diversification of hyperpolarization-activated cyclic nucleotide-gated channel genes. Physiol Genomics 29:231-245.

Johnston D, Wu SM (1995) Foundations of cellular neurophysiology. Cambridge, MA: MIT.

Kamiyama Y, Ogura T, Usui S (1996) Ionic current model of the vertebrate rod photoreceptor. Vision Res 36:4059-4068.

Kawai F, Horiguchi M, Ichinose H, Ohkuma M, Isobe R, Miyachi E (2005) Suppression by an $\mathrm{h}$ current of spontaneous $\mathrm{Na}+$ action potentials in human cone and rod photoreceptors. Invest Ophthalmol Vis Sci 46:390-397.

Kole MHP, Hallermann S, Stuart GJ (2006) Single $I_{\mathrm{h}}$ channels in pyramidal neuron dendrites: properties, distribution, and impact on action potential output. J Neurosci 26:1677-1687.

Liu XD, Kourennyi DE (2004) Effects of tetraethylammonium on kx channels and simulated light response in rod photoreceptors. Ann Biomed Eng 32:1428-1442.

Magee JC (1998) Dendritic hyperpolarization-activated currents modify the integrative properties of hippocampal CA1 pyramidal neurons. J Neurosci 18:7613-7624.

Malcolm AT, Kourennyi DE, Barnes S (2003) Protons and calcium alter 
gating of the hyperpolarization-activated cation current $(\mathrm{I}(\mathrm{h}))$ in rod photoreceptors. Biochim Biophys Acta 1609:183-192.

Männikkö R, Pandey S, Larsson HP, Elinder F (2005) Hysteresis in the voltage dependence of hon channels: conversion between two modes affects pacemaker properties. J Gen Physiol 125:305-326.

Maricq AV, Korenbrot JI (1990) Inward rectification in the inner segment of single retinal cone photoreceptors. J Neurophysiol 64:1917-1928.

Marmarelis PZ, Marmarelis VZ (1978) Analysis of physiological systems: the white-noise approach. In: Computers in biology and medicine (Moore GP, ed), pp 131-180. New York: Plenum.

Marmarelis PZ, Naka KI (1973) Nonlinear analysis and synthesis of receptive-field responses in the catfish retina. 3. Two-input white-noise analysis. J Neurophysiol 36:634-648.

Müller F, Scholten A, Ivanova E, Haverkamp S, Kremmer E, Kaupp UB (2003) Hcn channels are expressed differentially in retinal bipolar cells and concentrated at synaptic terminals. Eur J Neurosci 17:2084-2096.

Narayanan R, Johnston D (2007) Long-term potentiation in rat hippocampal neurons is accompanied by spatially widespread changes in intrinsic oscillatory dynamics and excitability. Neuron 56:1061-1075.

Ogura T, Satoh TO, Usui S, Yamada M (2003) A simulation analysis on mechanisms of damped oscillation in retinal rod photoreceptor cells. Vision Res 43:2019-2028.

Owen WG, Torre V (1983) High-pass filtering of small signals by retinal rods. Ionic studies. Biophys J 41:325-339.
Publio R, Oliveira R, Roque A (2006) A realistic model of rod photoreceptor for use in a retina network model. Neurocomputing 69:1020-1024.

Sakmann B, Neher E (1995) Single-channel recording, Ed 2. New York: Plenum.

Satoh TO, Yamada M (2000) A bradycardiac agent zd7288 blocks the hyperpolarization-activated current (ih) in retinal rod photoreceptors Neuropharmacology 39:1284-1291.

Sigworth FJ (1980) The variance of sodium current fluctuations at the node of Ranvier. J Physiol 307:97-129.

Siu CW, Lieu DK, Li RA (2006) Hcn-encoded pacemaker channels: from physiology and biophysics to bioengineering. J Membr Biol 214:115-122.

Stieber J, Stöckl G, Herrmann S, Hassfurth B, Hofmann F (2005) Functional expression of the human hen3 channel. J Biol Chem 280:34635-34643.

Stieber J, Wieland K, Stöckl G, Ludwig A, Hofmann F (2006) Bradycardic and proarrhythmic properties of sinus node inhibitors. Mol Pharmacol 69:1328-1337.

Torre V, Owen WG (1983) High-pass filtering of small signals by the rod network in the retina of the toad, Bufo marinus. Biophys J 41:305-324.

Wollmuth LP, Hille B (1992) Ionic selectivity of ih channels of rod photoreceptors in tiger salamanders. J Gen Physiol 100:749-765.

Zhang J, Wu SM (2005) Physiological properties of rod photoreceptor electrical coupling in the tiger salamander retina. J Physiol 564:849-862.

Zhang J, Wu SM (2009) Immunocytochemical analysis of photoreceptors in the tiger salamander retina. Vision Res 49:64-73. 